\title{
Endomyocardial Fibrosis Associated With Apical Calcification and High Uptake on Myocardial Gallium-67 Scintigraphy
}

Sonoko Maemura, MD; Eisuke Amiya, MD, PhD; Hikari Seki, MD; Kazutaka Ueda, MD, PhD;

Daisuke Nitta, MD; Teruhiko Imamura, MD, PhD; Masae Uehara, MD, PhD;

Takayuki Kawata, MD, PhD; Masafumi Watanabe, MD, PhD; Masaru Hatano, MD, PhD; Koichiro Kinugawa, MD, PhD; Issei Komuro, MD, PhD
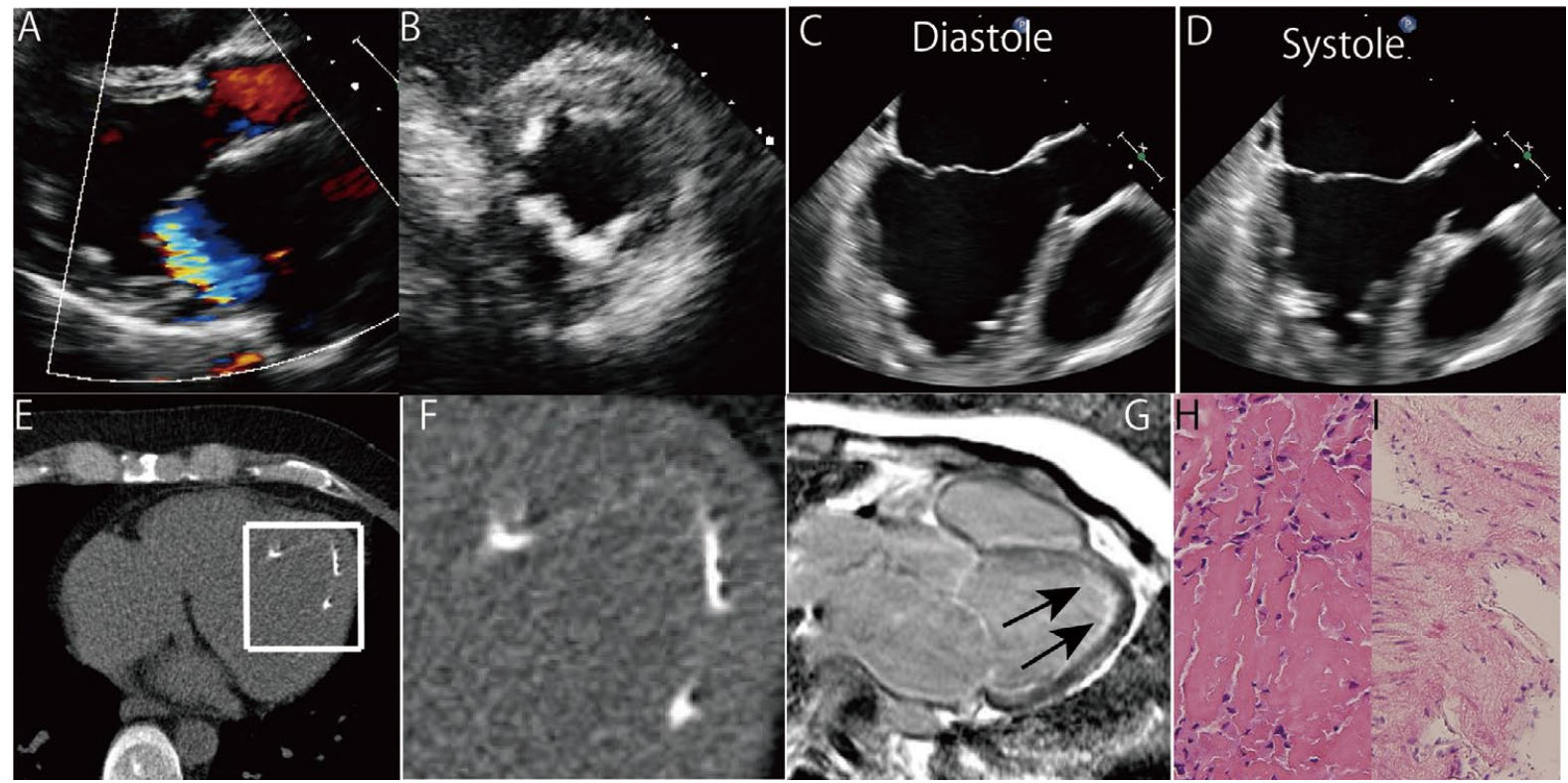

Figure 1. (A,B) Transthoracic echocardiogram (A, substernal long axis view). Severe mitral regurgitation due to annulus expansion was identified. (B) Substernal short axis view showed significant increase of apical endocardial echo density. (C,D) Transesophageal echocardiogram (apical view). Ventricular apical obliteration during (C) diastole and (D) systole. Apical endocardial echo density was significantly enhanced. (E,F) Electrocardiogram-gated computed tomography showing scattered calcification alongside the apical region of the endocardium ( $F$, magnified view). (G) Late gadolinium enhancement magnetic resonance imaging showed a characteristic pattern of high-signal intensity confined to the subendocardial area (black arrows) without any findings of thrombi. $(\mathbf{H}, \mathbf{I})$ Microscopy of $(\mathbf{H})$ left and $(\mathbf{I})$ right ventricular myocardium specimens.

$\mathbf{E}$ ndomyocardial fibrosis (EMF) is a common cardiovascular disorder in tropical countries, but it is an extremely rare disease in developed countries. ${ }^{1,2}$ Clinical features of EMF include restrictive cardiomyopathy and atrioventricular valve regurgitation because of the pres- ence of fibrotic tissue in the endomyocardium. Several causes of EMF have been suggested, including hypereosinophilia, parasitic infection and cerium exposure, but, the etiology is still unclear and the efficacy of therapeutic interventions has not been well established. ${ }^{3}$ In this study, we report a represen-

Received May 25, 2016; revised manuscript received July 5, 2016; accepted July 6, 2016; released online July 29, 2016 Time for primary review: 19 days

Department of Cardiovascular Medicine, Graduate School of Medicine, The University of Tokyo, Tokyo (S.M., E.A., H.S., K.U., D.N., T.I., M.U., T.K., M.W., M.H., I.K.); Second Department of Internal Medicine, University of Toyama, Toyama (K.K.), Japan

Mailing address: Eisuke Amiya, MD, PhD, Department of Cardiovascular Medicine, Graduate School of Medicine, The University of Tokyo, 7-3-1 Hongo, Bunkyo-ku, Tokyo 113-8655, Japan. E-mail: amiyae-tky@umin.ac.jp

ISSN-1346-9843 doi:10.1253/circj.CJ-16-0512

All rights are reserved to the Japanese Circulation Society. For permissions, please e-mail: cj@j-circ.or.jp 


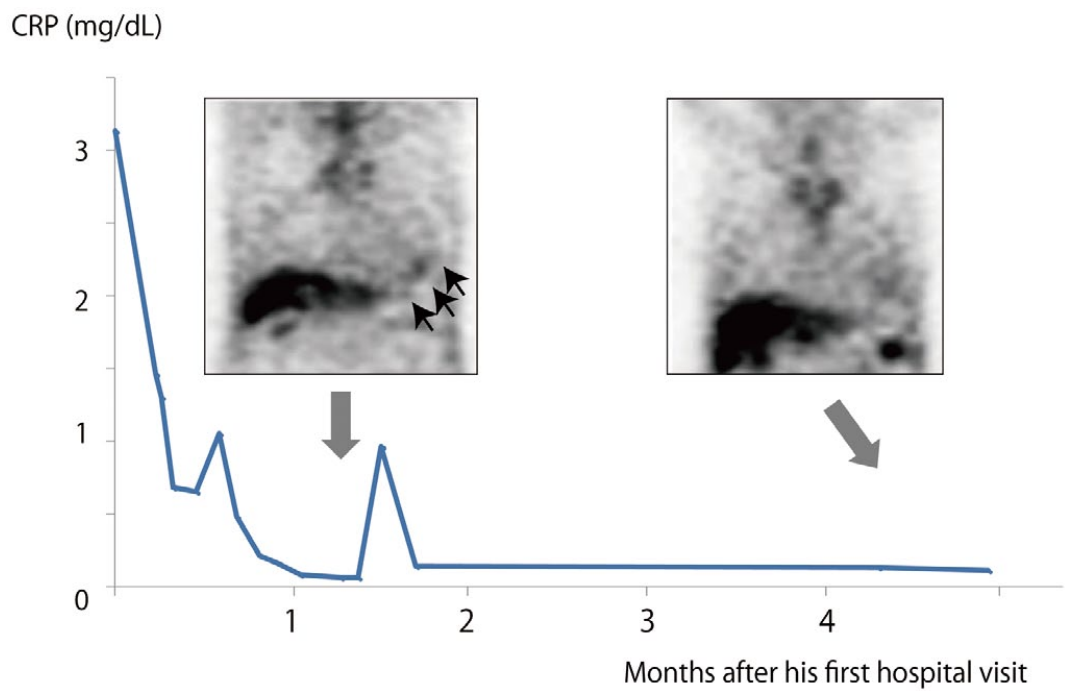

Figure 2. Myocardial gallium-67 scintigraphy showing high uptake at the endocardial apex. Decreased uptake at the endocardial apex, accompanied by spontaneous reduction in C-reactive protein over a 4-month period, suggested a fluctuating inflammatory response on the endocardial surface of the myocardium.

tative case of EMF with left ventricular inflammation visualized on myocardial gallium-67 scintigraphy.

The patient was a 37-year-old Japanese man who presented with dyspnea on exertion. Chest X-ray showed pulmonary edema and he was diagnosed with heart failure. Echocardiography showed moderate mitral regurgitation and severe diastolic dysfunction. Despite optimal medical therapy for 2 years, his New York Heart Association functional class remained IV, and he was referred to The University of Tokyo Hospital for further management. He had no previous history of significant illness and no family history of cardiac disorders. No eosinophilia was detected, and brain natriuretic peptide was $240.2 \mathrm{pg} / \mathrm{ml}$. Electrocardiogram showed sinus rhythm, left axis deviation and complete left bundle branch block. Repeat echocardiogram indicated moderate left atrial dilatation as well as mild left ventricular dilatation with severe diastolic dysfunction (septal E/e', 15.4). Severe mitral regurgitation due to annulus expansion was also identified (Figure 1A). In particular, the most remarkable finding on echocardiography was the significant increase of apical endocardial echo density, and this pathological change was complicated with ventricular apical obliteration during systole (Figures 1B-D). This endocardial abnormality corresponded to scattered severe calcifications alongside the apical endocardium on electrocardiogram-gated computed tomography (Figures 1E,F). Coronary angiography was normal, but cardiac index was reduced to $1.98 \mathrm{~L} / \mathrm{min} / \mathrm{m}^{2}$, with a mean pulmonary capillary wedge pressure of $27 \mathrm{mmHg}$. On cardiopulmonary exercise testing, maximum oxygen consumption was reduced to $14.6 \mathrm{ml} / \mathrm{kg} / \mathrm{min}$, and on exercise echocardiography pulmonary hypertension was significantly exacerbated in the absence of increased mitral regurgitation after submaximum exercise. This was suggestive of severely restricted exercise tolerance mainly derived from a severe impairment in diastolic function. Late gadolinium enhancement magnetic resonance imaging (MRI) showed a characteristic pattern of high-signal intensity confined to the subendocardial area without any findings of thrombi (Figure 1G). On histological work-up, specimens from the left ventricular myocardium had fibrin deposition with inflammatory infiltrates including histiocytes (Figure 1H), whereas specimens from right ventricular myocardium con- firmed EMF with atrophic cardiomyocyte (Figure 1I). On the basis of these clinical findings, EMF was diagnosed. Extended endomyocardectomy and atrioventricular valvuloplasty have previously been reported as effective therapeutic interventions, but they were not considered in this case because procedural complications were assessed as outweighing any potential benefit to the patient.,5 Therefore, we decided to continue medical therapy while applying for heart transplant.

The underlying pathological process in EMF involves the occurrence of progressive fibrosis around the endocardial surface of the left and right ventricles, resulting in restrictive physiology. This disease is associated with eosinophilia or parasitic infection, suggesting that inflammation is a critical step in the progression. ${ }^{6}$ To date, however, the early phase of EMF has not been well described, because most patients do not exhibit symptoms until they are at a late stage in the clinical course. ${ }^{7}$ Late gadolinium enhancement MRI is useful for detecting subendocardial fibrosis, but not for detecting active inflammation. ${ }^{8}$

In the current case, we checked the state of inflammation using gallium-67 scintigraphy, and noted high uptake at the ventricular apex as a sign of active inflammation (Figure 2). To our knowledge, this is the first use of gallium-67 scintigraphy in EMF. Using this technique, we also identified a fluctuating inflammatory reaction because the uptake on gallium-67 scintigraphy was found to be decreased 4 months after the first hospital visit, accompanied by spontaneous improvement in previously elevated C-reactive protein to within normal range (Figure 2). This possibly corresponded to the fluctuation of inflammation on the endocardial surface of the myocardium. F-18-fluorodeoxyglucose positron-emission tomography (FDGPET) may be more useful for the identification of the inflammation in EMF. 9 The identification of inflammation on gallium-67 scintigraphy or FDG-PET may enable early diagnosis and concise evaluation required to facilitate interventions to suppress the inflammatory reaction in EMF. ${ }^{10,11}$ This could eventually improve the prognosis of this disease.

\section{Disclosures}

The authors declare no conflicts of interest. 


\section{References}

1. Mocumbi AO, Ferreira MB, Sidi D, Yacoub MH. A population study of endomyocardial fibrosis in a rural area of Mozambique. $N$ Engl $J$ Med 2008; 359: 43-49.

2. Mocumbi AO, Falase AO. Recent advances in the epidemiology, diagnosis and treatment of endomyocardial fibrosis in Africa. Heart 2013; 99: 1481-1487.

3. Bukhman G, Ziegler J, Parry E. Endomyocardial fibrosis: Still a mystery after 60 years. PLoS Negl Trop Dis 2008; 2: e97, doi: 10.1371/journal.pntd.0000097.

4. Moraes F, Lapa C, Hazin S, Tenorio E, Gomes C, Moraes CR. Surgery for endomyocardial fibrosis revisited. Eur J Cardiothorac Surg 1999; 15: 309-312.

5. Schneider U, Jenni R, Turina J, Turina M, Hess OM. Long-term follow up of patients with endomyocardial fibrosis: Effects of surgery. Heart 1998; 79: 362-367.

6. Iglezias SD, Benvenuti LA, Calabrese F, Salemi VM, Silva AM, Carturan E, et al. Endomyocardial fibrosis: Pathological and molecular findings of surgically resected ventricular endomyocardium.
Virchows Arch 2008; 453: 233-241.

7. Hassan WM, Fawzy ME, Al Helaly S, Hegazy H, Malik S. Pitfalls in diagnosis and clinical, echocardiographic, and hemodynamic findings in endomyocardial fibrosis: A 25 -year experience. Chest 2005; 128: $3985-3992$.

8. Cury RC, Abbara S, Sandoval LJ, Houser S, Brady TJ, Palacios IF. Visualization of endomyocardial fibrosis by delayed-enhancement magnetic resonance imaging. Circulation 2005; 111: e115-e117, doi:10.1161/01.CIR.0000157399.96408.36.

9. Miyagawa M, Yokoyama R, Nishiyama Y, Ogimoto A, Higaki J, Mochizuki T. Positron emission tomography-computed tomography for imaging of inflammatory cardiovascular diseases: Sarcoidosis, large-vessel arteritis, and atherosclerosis. Circ J 2014; 78: $1302-$ 1310.

10. JCS Joint Working Group. Guidelines for diagnosis and treatment of myocarditis (JCS 2009): Digest version. Circ J 2011; 75: 734-743.

11. Yasui H, Takahama H, Kanzaki H, Ishibashi-Ueda H, Morita N, Sugano Y, et al. Time-course changes of cardiac-specific inflammation in a patient with left ventricular calcified amorphous tumor. Circ J 2015; 79: 2069-2071. 